\title{
MATERNAL FACTORS THAT ENHANCE THE ACQUISITION OF GROUP-B STREPTOCOCCI BY NEWBORN INFANTS
}

\author{
R. J. Ancona, Patricia Ferrieri and P. P. Williams \\ Departments of Pediatrics and Obstetrics, University of Minnesota \\ Medical School, Minneapolis, Minn. 55455, USA
}

MOST INVESTIGA TORS agree that in the early-onset form of neonatal group-B streptococcal disease the infecting micro-organism is acquired from the maternal genital tract (Harper, 1971; Baker and Barrett, 1973; Franciosi, Knostman and Zimmerman, 1973; Anthony and Okada, 1977; Ferrieri, Cleary and Seeds, 1977; Anthony, Okada and Hobel, 1978). Prematurity, prolonged rupture of the fetal membranes, and other obstetric complications are more frequent in colonised infants who develop disease than in those who do not (Baker and Barrett, 1973; Franciosi et al., 1973). None of these factors influences the development of late-onset group-B streptococcal disease, in which nosocomial transmission may be of more importance (Steere et al., 1975; Aber et al., 1976).

The factors that influence colonisation of neonates are not well understood. In a few studies in which the sex, race, length of gestation and birth weight of asymptomatic colonised and non-colonised infants were compared, no differences were found (Baker and Barrett, 1973; Aber et al., 1976; Baker, 1977). Some infants who do not yield group-B streptococci when swabbed immediately after birth have acquired them by the time of discharge from the hospital (Steere et al., 1975; Aber et al., 1976; Ferrieri et al., 1977). Whether they are at the same risk as infants colonised at birth of developing early or late-onset disease is not known. If high rates of neonatal colonisation favour an increased incidence of disease (Regan et al., 1979), and because $28-72 \%$ (Aber et al., 1976; Baker, 1977; Regan et al., 1979) of infants born to mothers with positive genital cultures acquire group-B streptococci, it is important to identify factors that might favour the transmission of the organisms from mother to infant. We have attempted to do this in a prospective study of a population of mothers and their newborn infants.

\section{MATERIALS AND METHODS}

Women who delivered infants at the University of Minnesota Hospitals from 1 Aug. 1976 to 1 Nov. 1978, and their infants, were included in the investigation. Swabs were taken from the vagina of the women, from the anterior nares, external ear canals and base of the umbilicus of the infants $2-4 \mathrm{~h}$ after birth, and again from the anterior nares and base of the umbilicus at the time

Received 20 Aug. 1979; accepted 2 Oct. 1979.

Requests for reprints to Dr Patricia Ferrieri. Box 134 Mayo, University of Minnesota, Minneapolis, Minn. 55455 . 
of discharge from hospital (usually $c .5$ days later). Additional cultures were made at the discretion of the attending physicians.

Swabs were collected and examined as described by Ferrieri et al. (1977). Briefly, they were plated on $6 \%$ sheep-blood agar containing colistin $10 \mathrm{mg} /$ litre and nalidixic acid $15 \mathrm{mg} /$ litre (Columbia CNA Agar, Difco Laboratories, USA). Colonies of group-B streptococci were identified by their colonial appearances and enumerated. All isolates were grouped and typed serologically. The heaviness of growth on the primary plates was scored as follows: $1+=1-10$ colonies, $2+=11-50$ colonies, $3+=>50$ colonies.

The charts of all mother-infant pairs from whom any culture was positive for group-B streptococci during the study period were reviewed. Control mother-infant pairs with negative streptococcal cultures were selected by age-matching of infants born within 1 day of the study infants.

The women who took part in the study had previously given written permission for cultures to be taken from them and their infants, and the protocol for the study had been approved by the Committee for the Use of Human Subjects in Research, University of Minnesota.

\section{RESULTS}

Isolation of group-B streptococci in the entire study population. During the 27-month study period, cultures were made from 1416 women in labour, of whom $113(8 \%)$ yielded group-B streptococci. Cultures were made from all 1675 infants born during this period; $57(3.4 \%)$ of the infants yielded at least one positive culture at birth, and $98(5.9 \%)$ at birth, at discharge from hospital, or both. The culture status of the mother was known for 43 of the 57 infants who were culture positive at birth; 38 mothers were culture positive and five culture negative in labour.

Enumeration of maternal cultures and neonatal acquisition of streptococci. Semi-quantitative culture data were available for the 113 mothers who had positive vaginal cultures in labour. In all, $38(34 \%)$ of the 113 infants had positive cultures at birth and $23(20 \%)$ had positive cultures for the first time at discharge; thus, the total number of acquisitions was $61(54 \%)$. At the time of discharge, 43 infants $(38 \%)$ gave positive swabs.

The frequency of acquisition of group-B streptococci by the infants was directly related to the heaviness of vaginal carriage in the mother during labour. Thus, the total acquisition rate was $44 / 66(67 \%)$ for infants born to mothers with $3+$ cultures but only $17 / 47(36 \%)$ for those born to mothers with $2+$ or $1+$ cultures $(p<0.005)$. For colonisation at birth (see the figure), the rates for infants of $3+, 2+$ and $1+$ mothers were respectively $48 \%, 24 \%$ $(0.05<\mathrm{p}<0 \cdot 10)$ and $4 \%(\mathrm{p}<0 \cdot 005)$.

Colonised infants born to mothers with a $3+$ vaginal culture were more often positive at birth $(32 / 44,73 \%)$ than were colonised infants born to $1+$ and $2+$ mothers $(6 / 17,35 \% ; p<0.02)$. Nearly one-half $(11 / 23)$ of all infants with positive swabs for the first time at discharge were born to mothers with $2+$ or $1+$ swabs.

Among the infants who gave positive cultures at birth, 19/32 (59\%) born to $3+$ mothers, but only $1 / 6$ born to $2+$ or $1+$ mothers, gave positive cultures at the time of discharge. Of the total of 43 infants who gave positive cultures at 


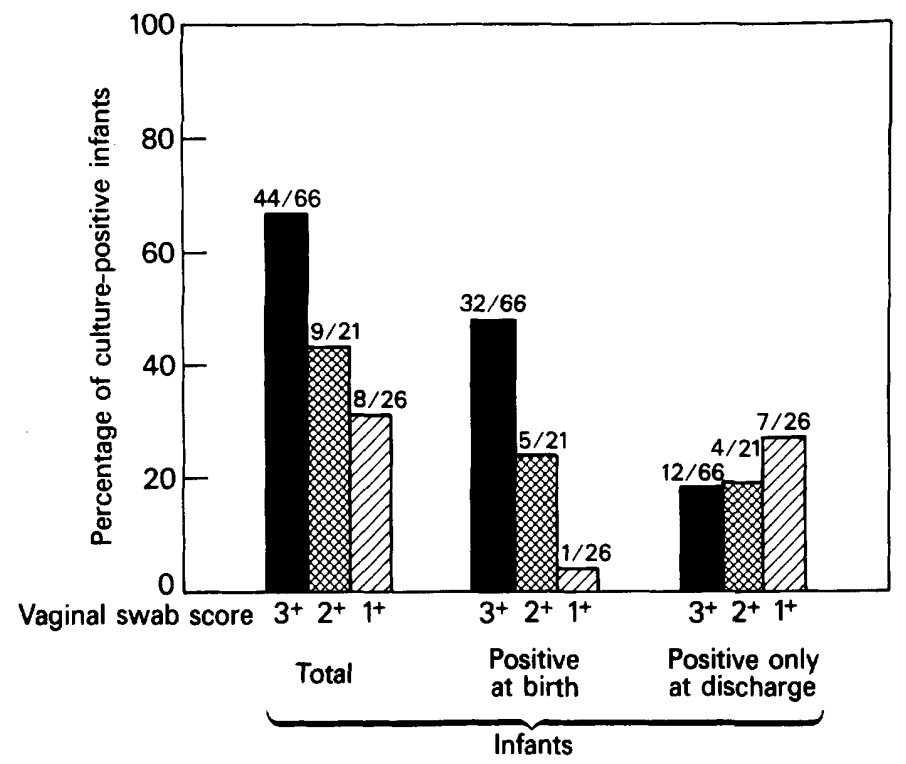

FigURE.-Frequency of acquisition of group-B streptococci by infants of mothers with positive vaginal swabs during labour: at birth, for the first time at discharge from hospital, and the sum of these ("total"), in relation to the heaviness of growth from the vaginal swab $(3+=>50,2+=11-50$, and $1+=1-10$ colonies). Figures above bars: number of positive infants born to numbers of mothers with the designated culture result.

discharge, $31(72 \%)$ were born to $3+$ mothers but only $12(28 \%)$ were born to $2+$ or $1+$ mothers $(\mathrm{p}<0 \cdot 02)$.

There were 38 mother-infant pairs in which the mother had given a positive vaginal culture in labour and group-B streptococci were isolated at birth from one or more skin or mucosal site in the corresponding infant; in 32 of these $\left(84^{\circ}\right)$ ) the mother's vaginal culture was heavy $(3+)$. Of the 38 colonised infants, $22(58 \%)$ gave positive cultures from two or more and $14(37 \%)$ from three or more sites. Of these last, $12 / 14$ had been born to mothers with $3+$ vaginal cultures. The likelihood that an infant would be heavily contaminated with group-B streptococci was also influenced by the degree of colonisation of the mother's genital tract. Of 19 infants with a $3+$ culture from at least one skin or mucosal site at birth, $18\left(95^{\circ} \%\right)$ were born to mothers with $3+$ vaginal cultures.

Duration of labour after rupture of the membranes (LRM) and neonatal acquisition of group-B streptococci. Infants of mothers with a positive vaginal swab in labour and a duration of LRM exceeding $6 \mathrm{~h}$ (table I) were somewhat more often colonised at birth $(18 / 38 ; 47 \%)$ than were infants of mothers with a positive swab but a shorter duration of LRM $(20 / 75 ; 27 \%$; $<<0.05)$. However, no difference in the eventual rate of acquisition (at birth + at discharge) was observed: $\mathrm{LRM}>6 \mathrm{~h}, 22 / 38(58 \%)$; LRM $<6 \mathrm{~h}, 39 / 75(52 \%$, p $>0.50)$. In all, 22/61 infants $(36 \%)$ positive at birth, at discharge, or both, and $16 / 52$ culture-negative infants $(31 \%)$, had been born to mothers with a positive vaginal swab $>6 \mathrm{~h}$ after rupture of the membranes $(p>0 \cdot 50)$. The results 
TABLE I

Duration of labour after rupture of the membranes (LRM) and the acquisition of group-B streptococci by neonates; duration of $L R M$ in women with positive vaginal swab and in matched controls with a negative swab

\begin{tabular}{|c|c|c|c|c|c|}
\hline \multirow[b]{4}{*}{$\begin{array}{l}\text { Duration } \\
\text { of LRM }\end{array}$} & \multicolumn{5}{|c|}{ Number of women with the stated duration of LRM and a } \\
\hline & \multicolumn{4}{|c|}{ positive vaginal swab } & \multirow{3}{*}{$\begin{array}{c}\text { negative } \\
\text { vaginal } \\
\text { swabt }\end{array}$} \\
\hline & \multirow[t]{2}{*}{ Total } & \multicolumn{3}{|c|}{ whose infants' swabs were } & \\
\hline & & $\begin{array}{l}\text { positive } \\
\text { at birth }\end{array}$ & $\begin{array}{c}\text { positive } \\
\text { at any } \\
\text { time* }\end{array}$ & negative & \\
\hline $\begin{array}{l}<6 \mathrm{~h} \\
>6 \mathrm{~h} \\
\text { Either }\end{array}$ & $\begin{array}{r}75 \\
38 \\
113\end{array}$ & $\begin{array}{l}20 \\
18 \\
38\end{array}$ & $\begin{array}{l}39 \\
22 \\
61\end{array}$ & $\begin{array}{l}36 \\
16 \\
52\end{array}$ & $\begin{array}{r}73 \\
40 \\
113\end{array}$ \\
\hline
\end{tabular}

* A positive result at birth, at discharge from hospital, or both.

+ For selection of controls, see Materials and methods.

were not changed by the inclusion of data on 19 infants who were culture positive at birth but whose mothers' culture status was negative (five) or unknown (14). There was no difference in length of labour after rupture of the membranes between culture-negative control mothers and mothers with positive streptococcal cultures in labour (table I).

Comparison of study and control mother-infant pairs. The control motherinfant pairs did not differ from the study mother-infant pairs in maternal age, number of pregnancies, infant birth weight or gestational age (table II). Although a somewhat higher percentage of mothers in the study group had postpartum fever and received antibiotics than in the control group, this difference was not statistically significant. Of the 17 colonised mothers with fever or post partum infections, or both, eight had been in labour with ruptured membranes for $>6 \mathrm{~h}$. In five of these 17 mothers $(29 \%)$, the membranes had

TABLE II

Comparison of the study group of mother-infant pairs (mother a carrier of group-B streptococci during labour) with a group of matched control pairs (mother a non-carrier)

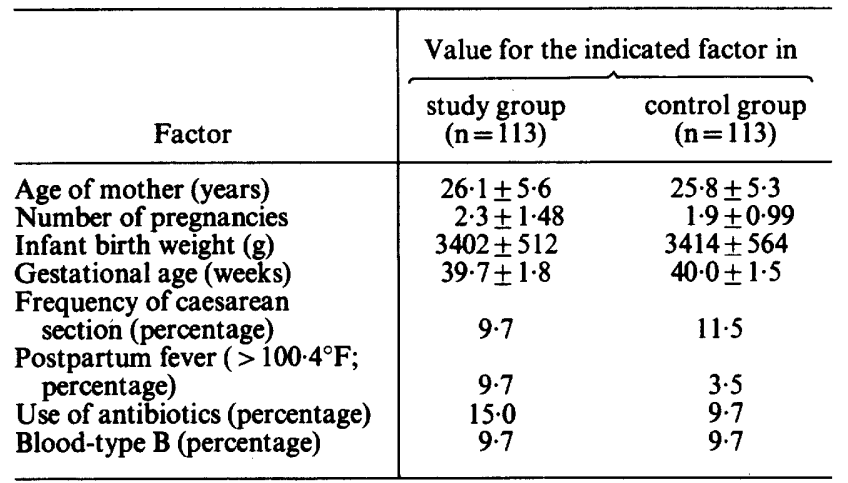


TABLE III

Distribution of serotypes among group-B streptococcal isolates from mothers and infants

\begin{tabular}{|c|c|c|c|c|}
\hline \multirow[t]{3}{*}{ Serotype } & \multicolumn{3}{|c|}{$\begin{array}{l}\text { Number (and percentage) of } \\
\text { isolates of the stated serotype from }\end{array}$} & \\
\hline & \multirow{2}{*}{$\begin{array}{l}\text { vaginal swabs } \\
\text { of mothers }\end{array}$} & \multicolumn{3}{|c|}{ infants } \\
\hline & & at birth & at discharge* & Total \\
\hline Ia & $3(3)$ & 1 & 1 & $2(3)$ \\
\hline $\mathrm{Ib}$ & $23(20)$ & 7 & 3 & $10(16)$ \\
\hline Ic & $27(24)$ & 8 & 7 & $15(25)$ \\
\hline II & $32(28)$ & 6 & 8 & $14(23)$ \\
\hline III & $25(22)$ & $13(34)$ & 4 & $17(28)$ \\
\hline NT & $3(3)$ & 3 & 0 & $3(5)$ \\
\hline Any & 113 & 38 & 23 & 61 \\
\hline
\end{tabular}

* Includes only infants positive for the first time at discharge. $\mathrm{NT}=$ not typable.

been ruptured for $>12 \mathrm{~h}$, in comparison with 11 of the $96(11 \%)$ other colonised mothers $(p>0 \cdot 10)$.

An association between blood-group B and increased frequency of vaginal carriage of group-B streptococci has been suggested (Regan, Chao and James, 1978). We found a similar distribution of blood types in maternal carriers of group-B streptococci and control mothers; blood-type B occurred in $c .10 \%$ of our control and study mothers.

Streptococcal serotypes in mothers and infants. The serotypes of the group-B streptococci isolated from vaginal cultures obtained in labour and from cultures taken from the infants at birth or discharge were similar (table III). Serotypes Ic, II and III were the most prevalent. However, $52 \%$ of mothers carrying serotype-III group-B streptococci delivered infants colonised at birth with strains of this serotype; the corresponding rate for mothers harbouring all other serotypes was $28 \%(25 / 88 ; p<0.05)$. However, there was no significant difference in the predominance of any single serotype when the cumulative acquisition rates (infants positive at birth or discharge) were compared.

\section{DisCUSSION}

An $8 \%$ vaginal carriage rate of group-B streptococci in this group of pregnant women in labour was comparable with earlier findings in this hospital (Ferrieri et al., 1977). Other workers have recorded carriage rates as low as $5 \%$ and as high as $28 \%$ (Reid, 1975; Anthony and Okada, 1977; Baker, 1977); these differences may reflect uneven geographical distribution of the organisms, as well as the number of body sites cultured, the frequency of sampling, and whether or not selective media were used. Our population has a low frequency of gonococcal carriage and infection and a low proportion of teenage pregnancies (Ferrieri et al., 1977). 
Our results showed that the number of group-B streptococci in the vagina during labour is a major determinant for mother-infant transmission, as measured not only by the proportion of infants from whom the organisms could be isolated soon after birth, but also by the number of surface sites contaminated and the heaviness of this contamination. The use of enrichment broth cultures containing antibiotics may increase the numbers of positive genital-tract cultures for group-B streptococci (Mason, Wong and Barrett, 1976), but their exclusive use does not distinguish between heavily and lightly colonised women.

Prolonged labour after rupture of the fetal membranes has been associated with early onset group-B streptococcal disease (Anthony and Okada, 1977; Baker, 1977). The proposed pathogenesis is through an ascending infection that results in extended contact of the fetus with the organisms (Wannamaker and Ferrieri, 1975). In the present study, labour for $>6 \mathrm{~h}$ after rupture of the membranes was associated with an increased rate of initial contamination of the infant, but had no significant effect on the total rate of acquisition of group-B streptococci. This factor may be of importance when the mother's vagina is only lightly colonised, because four of six infants who were born to the $1+$ or $2+$ mothers and had positive cultures at birth had been delivered $>6 \mathrm{~h}$ after rupture of the membranes. Most infants born to colonised mothers $(66 \%)$ and to controls $(65 \%)$ had been delivered $<6 \mathrm{~h}$ after rupture of the membranes. There was no significant difference in the duration of labour after rupture of the membranes between colonised and non-colonised mothers.

Culture-positive mothers in labour did not differ significantly from controls in any of the other variables examined. A higher percentage of the colonised than the non-colonised mothers developed postpartum fever and required antibiotics; and the culture-positive mothers who had fever, postpartum infection, or both, had been in labour for $>6 \mathrm{~h}$ after rupture of the membranes twice as often as colonised mothers without these complications, but this difference was not significant. Prolonged labour after rupture of the membranes is known to increase the rate of postpartum complications (Blanc, 1961; Reid, 1975). Whether vaginal colonisation with group-B streptococci increases this risk for the mother is not clear from the available data.

Regan et al. (1978) reported an excess of patients of blood-group B among women colonised with group-B streptococci, but our observations did not confirm this finding. The populations studied were different, but the frequency of blood-type B in our population was lower than in any of these workers' subgroups, including their culture-negative private patients. More data are needed to explore this possible association.

The distribution of group-B streptococcal serotypes in our mothers and infants was similar to that previously reported in the United States (Baker and Barrett, 1973, 1974; Anthony and Okada, 1977; Ferrieri et al., 1977; Pass et al., 1979). Mothers carrying type-III group-B streptococci delivered infants positive at birth significantly more often than did mothers carrying all other serotypes, but when infants colonised at discharge were also considered the difference was no longer present. The increased prevalence of serotype III in 
infants at birth might represent some biological advantage of serotype III, such as increased adherence to epithelial cells, but there is no evidence at present to support this possibility.

\section{SUMMARY}

Bacteriological and clinical data in respect of 113 mothers who were vaginal carriers of group-B streptococci at the time of labour, and of their 113 infants, were examined for factors that contributed to mother-infant transmission of these micro-organisms. A semi-quantitative system $(1+=1-10$, $2+=11-50$, and $3+=>50$ colonies) was used to record the heaviness of growth in all positive primary cultures.

Positive cultures at birth or at the time of discharge from hospital (or both) were obtained from $44 / 66$ of the infants of mothers with $3+$ vaginal cultures $(67 \%)$, but from only $17 / 47$ of the infants of mothers with $2+$ or $1+$ cultures $(36 \% ; \mathrm{p}<0.005)$. Of 38 infants who gave positive cultures at birth, $32(84 \%)$ were born to mothers with $3+$ vaginal cultures. At birth, infants born to heavily colonised mothers were more often positive at multiple sites and were more heavily contaminated than infants born to lightly colonised mothers. However, nearly one-half of the infants whose swabs were first positive on discharge were born to mothers with $2+$ or $1+$ swabs.

When the mothers had been in labour for $>6 \mathrm{~h}$ after rupture of the membranes, the proportion of infants yielding positive cultures at birth was higher $(47 \%)$ than the proportion for infants of mothers in labour for a lesser time after membrane rupture $(27 \% ; \mathrm{p}<0.05)$, but there was no difference between the two groups in the eventual isolation rate (at birth + at discharge).

Although the distribution of group-B streptococcal serotypes was similar for mothers and infants, mothers carrying serotype-III strains delivered infants positive at birth more frequently than did mothers carrying all other serotypes.

The most important predictive factor determining neonatal acquisition of group-B streptococci appeared to be the number of group-B streptococci in the maternal vagina at the time of labour.

This work was supported by a grant AI-13926 from the US Public Health Service. Dr Ancona was supported as a post-doctoral fellow on an NIH training grant AI-07054. We thank JoAnn Nelson, Joanne Kumagai, and Margaret Ragan for their technical assistance and Mary Ryan for preparation of the manuscript.

\section{REFERENCES}

Aber, R. C., Allen, N., Howell, J. T., Wilkinson, H. W. and Facklam, R. R. 1976. Nosocomial transmission of group B streptococci. Pediatrics, 58, 346.

Anthony, B. F. AND OKADA, D. M. 1977. The emergence of group B streptococci in infections of the newborn infant. A. Rev. Med., 28, 355 .

Anthony, B. F., OKada, D. M. AND Hobel, C. J. 1978. Epidemiology of group B Streptococcus: longitudinal observations during pregnancy. J. infect. Dis., 137, 524 .

BAKER, C. J. 1977. Summary of the workshop on perinatal infections due to group B Streptococcus. J. infect. Dis., 136, 137. 
BAKER, C. J. AND BARRETT, F. F. 1973. Transmission of group-B streptococci among parturient women and their neonates. J. Pediat., 83, 919.

BAKer, C. J. AND BarRetT, F. F. 1974. Group-B streptococcal infections in infants. The importance of the various serotypes. J. Am. med. Ass., 230, 1158.

BLANC, W. A., 1961. Pathways of fetal and early neonatal infection. Viral placentins, bacterial and fungal chorioaminionitis. J. Pediat., 59, 473.

Ferrieri, P., Cleary, P. P. and Seeds, A. E. 1977. Epidemiology of group-B streptococcal carriage in pregnant women and newborn infants. J. med. Microbiol., 10, 103.

Franciosi, R. A., Knostman, J. D. and Zimmerman, R. A. 1973. Group-B streptococcal neonatal and infant infections. J. Pediat., 82, 707.

HARPER, I. A. 1971. The importance of group B streptococci as human pathogens in the British Isles. J. clin. Path., 24, 438.

MASON, E. O., JR, WONG, P. AND BARRETT, F. F. 1976. Evaluation of four methods for detection of group-B streptococcal colonization. J. clin. Microbiol., 4, 429.

Pass, M. A., Gray, B. M., Khare, S. and Dillon, H. C., JR. 1979. Epidemiological studies of group-B streptococcal infections in neonates. In Pathogenic streptococci, edited by M. T. Parker. Reedbooks: Chertsey, p. 175.

Regan, J. A., ChaO, S. AND JAMES, L. S. 1978. Maternal ABO blood group type B: a risk factor in the development of neonatal group-B streptococcal disease. Pediatrics, $62,504$.

Regan, J. A., O'Neill, J., ChaO, S. AND JAmes, L. S. 1979. Maternal vs infant colonization: which better predicts group-B streptococcal disease? Pediat. Res., 13, 393.

REID, T. M. S. 1975. Emergence of group B streptococci in obstetric and perinatal infections. Br. med. J., 2, 533.

Steere, A. C., Aber, R. C., Warford, L. R., Murphy, K. E., Feeley, J. C., Hayes, P. S., Wilkinson, H. W. AND F ACKLAM, R. R. 1975. Possible nosocomial transmission of groupB streptococci in a newborn nursery. J. Pediat., 87, 784.

W ANNAMAKER, L. W. AND FerRIERI, P. 1975. Streptococcal infections-updated. In Disease-aMonth, October, edited by H. F. Dowling. Yearbook Medical Publishers: Chicago, p. 26. 